\title{
ANALYSIS OF THE INFLUENCE OF THE DIGGING POSITION ON THE LOADING OF THE SLEWING PLATFORM BEARING IN HYDRAULIC EXCAVATORS
}

\author{
Vesna Jovanović, Dragoslav Janošević, Jovan Pavlović \\ University of Niš, Faculty of Mechanical Engineering, Serbia
}

\begin{abstract}
The paper defines the general mathematical model of hydraulic excavators for determining the boundary and possible digging resistances and equivalent loads of the axial bearing of the slewing platform drive mechanism throughout the excavator's working area. Using a developed mathematical model and program, in the case of a $100000 \mathrm{~kg}$ hydraulic excavator with a shovel manipulator bucket volume of $6,5 \mathrm{~m}^{3}, a$ detailed analysis was performed to examine the influence of the position and digging resistance on the loading of the axial bearing of the excavator slewing platform drive mechanism. The results of the performed analysis show that the equivalent loads, relevant for the selection of axial bearing of the excavator slewing platform, occur in the zone of the working area when the kinematic chain of the excavator has positions in which the manipulator mechanisms have coordinated interaction when they can overcome the greatest resistance forces in the stable operation of the excavator.
\end{abstract}

Key Words: Hydraulic Excavator, Slewing Platform, Loading of Axial Bearing

\section{INTRODUCTION}

In the synthesis of the slewing platform drive mechanism of hydraulic excavators, one first chooses the axial bearing, which attaches the slewing platform to the support and movement mechanism of the excavator. The choice of this bearing is very complex because the excavator performs various functions in its working area with a variety of different positions and in very different working conditions. The recent research on the slewing platform drive mechanisms of excavators includes: development of general mathematical models of excavator [1-4], analysis of loading of large diameter axial bearings [5-7], the study of the influence factors on the bearing loads [8-11], the synthesis of the slewing platform drive mechanism [12] and the development of hybrid drives of the slewing platform drive mechanism which enables the energy recovery that occurs

Received February 25, 2019 / Accepted May 05, 2019

Corresponding author: Vesna Jovanović

University of Niš, Faculty of Mechanical Engineering, Serbia, Aleksandra Medvedeva 14, 18000 Niš, Serbia

E-mail: vesna.jovanovic@masfak.ni.ac.rs 
when the platform slews [13-15]. Within the research conducted by Jovanovic [12], general mathematical models and programs have been developed for determining the spectra of equivalent loads of the axial bearing of the slewing platform drive mechanisms in the entire working area of hydraulic excavators. By comparing the load spectra with the allowed loads, the size of the axial bearing can be reliably chosen. This paper presents the research results that show in which working area - i.e. the position of the kinematic chain of the excavator, and under which conditions of operation - the resistance of digging, the equivalent loads occur in the load bearing spectrum, for the choice of the size of the bearing of the slewing platform drive mechanism. During the research, a new method of analyzing the loading of the axial bearing of the slewing platform of hydraulic excavators was used, using the load spectra determined for the entire working area of the excavator according to the boundary forces that can overcome the drive mechanisms of the excavator and to the boundary forces that allow the stability of the excavator.

\section{MATHEMATICAL MODEL}

The research uses the general mathematical model of the excavator developed according to the physical model of the excavator with the support and movement mechanism $L_{1}$ (Fig. 1a), slewing platform $L_{2}$ and the manipulator with a boom $L_{3}$, stick $L_{4}$ and shovel bucket $L_{5}$. The support and movement mechanism and the slewing platform of the excavator are linked with a slewing joint in the form of an axial bearing. The slewing platform drive mechanism consists of a hydraulic motor with a planetary gearbox with a gear on the output shaft coupled with a toothed axial bearing. The actuators of the drive mechanisms of the excavator manipulator are two-way hydraulic cylinders of boom $c_{3}$, stick $c_{4}$ and bucket $c_{5}$. The numerical and experimental research [12] of the load of the axial bearing of the slewing platform drive mechanism have shown that: a) the greatest axial bearing loads in the digging operation of the excavator and b) the dynamic loads of the bearing, caused by the moving of the kinematic chain of the excavator in relation to the static loads, are relatively small bearing in mind that the digging process takes place slowly. Taking into account the results of the previous research on the loading of excavator axial bearing, a mathematical model of the excavator was developed with the following assumptions: 1) the support surface and the members of the kinematic chain of the excavator are modeled as rigid bodies; 2) during the manipulative task the excavator is subjected to external (technological) forces - digging resistance $\boldsymbol{W}$ and the gravitational force (weight) of: the members of the kinematic chain, the members of the drive system and the land grabbed by the bucket of the excavator; 3 ) during the digging operation, the kinematic chain of the excavator with a planar configuration is viewed as an open configuration chain whose last member - the bucket, is subjected to the possible digging resistance at the cutting edge of the bucket [16]. In analyzing the loading of the axial bearing of the slewing platform drive mechanism, the parameters of the kinematic chain members and the parameters of the drive mechanisms of the excavator are known.

\subsection{The space of the excavator model}

The space of the excavator model is determined by absolute coordinate system $O X Y Z$ with unit vectors $\vec{i}, \vec{j}, \vec{k}$ along coordinate axes $O X, O Y$ and $O Z$. The excavator support 
surface lies in the horizontal axis of the $O X Z$ absolute coordinate system, while vertical axis $O Y$ of the same system falls on the axis of the support and movement member-slewing member kinematic pair when the excavator is positioned on the horizontal surface. The internal (generalized) coordinates of the mathematical model of the kinematic chain of the excavator are angles $\theta_{i}$ (Fig. 1a) of the relative position of member $L_{i}$ in relation to previous member $L_{i-1}$ at rotation around the axis of joint $O_{i}$.

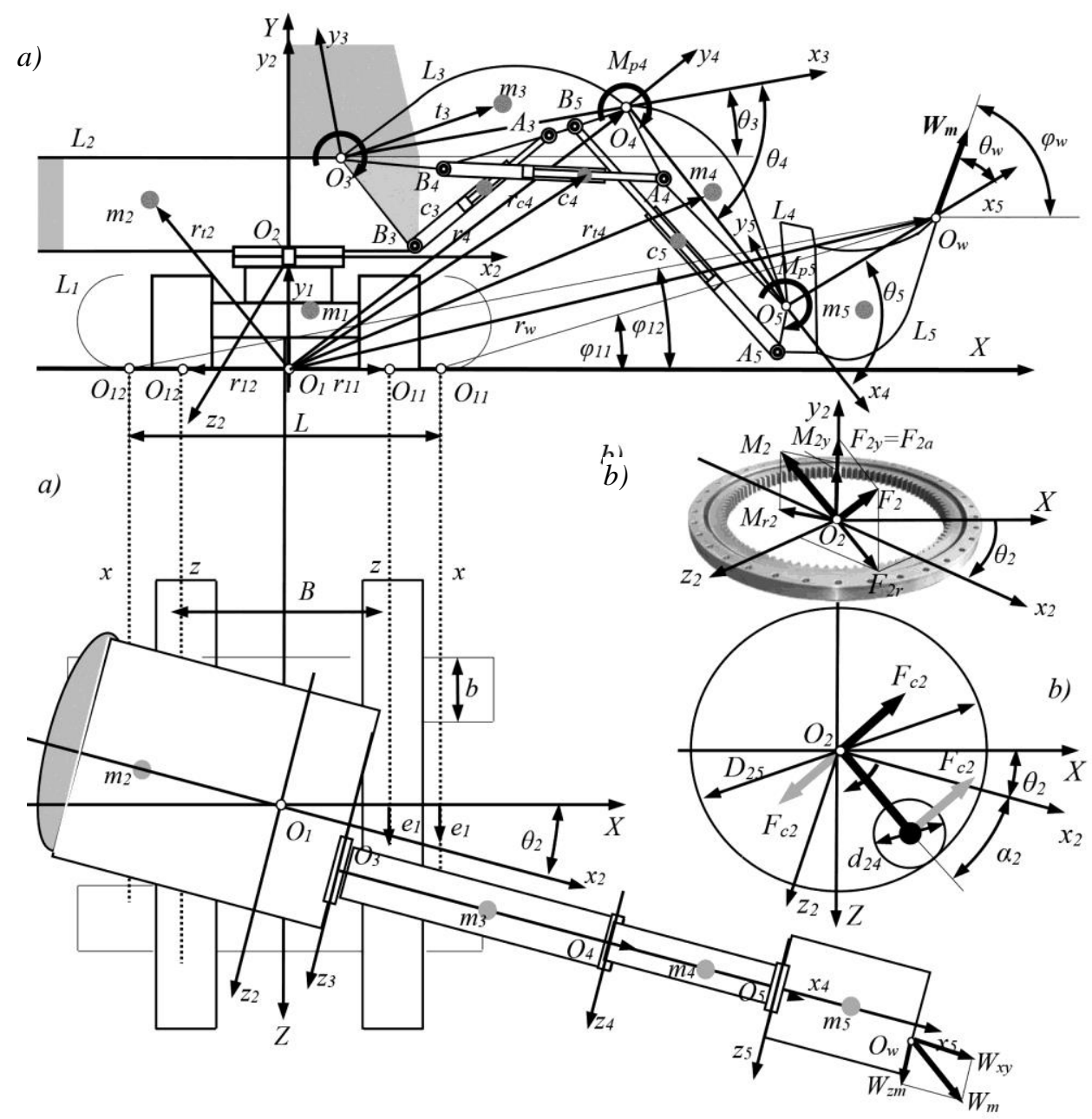

Fig. 1 Axial bearing load: a) mathematical model of excavator with a loading manipulator, b) load of slewing platform drive mechanism

By changing length $c_{i}$ of the hydraulic cylinders of the drive mechanisms in the interval of limit values $c_{i}=\left[c_{i p}, c_{c k}\right]$, generalized coordinates $\theta_{i}$ are changed in interval $\theta_{i}$ $=\left[\theta_{i p}, \theta_{i k}\right]$, where $\theta_{i p}$ is the initial and $\theta_{i k}$ is the final angle of the relative position of member $L_{i}$ to previous member $L_{i-1}$. The relative angle of movement $\theta_{i o}$ of member $L_{i}$ in relation to previous member $L_{i-1}$ is expressed by the difference: 


$$
\boldsymbol{\theta}_{i o}=\boldsymbol{\theta}_{i k}-\boldsymbol{\theta}_{i p}
$$

The position of member $\mathrm{Li}$ in relation to the horizontal $O X Z$ plane of the absolute coordinate system is determined by the angle:

$$
\boldsymbol{\varphi}_{i}=\sum_{i=3}^{i} \boldsymbol{\theta}_{i} \quad \forall i=3,4,5
$$

Vectors: $\vec{r}_{i}$ - the center of joint Oi, $\vec{r}_{t i}$ - the center of masses of the kinematic chain members and $\vec{r}_{w}$ - the center of the cutting edge of the bucket, are defined, in the absolute coordinate system, by the equations:

$$
\overrightarrow{\boldsymbol{r}}_{i}=\sum_{i=1}^{i-1} A_{i o} \overrightarrow{\boldsymbol{s}}_{i} \quad \forall i=2,3,4,5
$$

where: $A_{i o}$ - the transfer matrix used to transfer the vector quantities from local coordinate system $O_{i} x_{i} y_{i} z_{i}$ of member $L_{i}$ to absolute coordinate system $O X Y Z$

\subsection{Axial bearing load}

According to the defined mathematical model, force $F_{2}$ and moment $M_{2}$ of the load of the axial bearing of the excavator slewing platform drive mechanism are determined by the equations $[12,16]$ :

$$
\begin{gathered}
\boldsymbol{F}_{02}=\boldsymbol{g} \sum_{i=2}^{5} m_{i}+\sum_{i=3}^{5} m_{c i} \boldsymbol{g}+\boldsymbol{F}_{c 2}+\boldsymbol{W}_{m} \\
\boldsymbol{M}_{02}=\sum_{i=2}^{5} m_{i}\left(\left(\boldsymbol{r}_{t i}-\boldsymbol{r}_{2}\right) \times \boldsymbol{g}\right)+\sum_{i=3}^{5} m_{c i}\left(\left(\boldsymbol{r}_{c t i}-\boldsymbol{r}_{2}\right) \times \boldsymbol{g}\right)+\left(\boldsymbol{r}_{w}-\boldsymbol{r}_{2}\right) \times \boldsymbol{W}_{m}
\end{gathered}
$$

where: $m_{i}$ - member mass, $m_{c i}$ - mass of the actuators of the manipulator drive mechanisms, $F_{c 2}$ - reaction force of the slewing platform, and $W_{m}$ - possible force of digging resistance.

The reaction force of the slewing platform drive mechanism with one slewing drive is determined by the equation (Fig. 1b):

$$
\boldsymbol{F}_{c 2}=\frac{2\left|M_{2 y}\right|}{D_{25}-d_{24}}\left[\sin \left(\boldsymbol{\theta}_{2}+\boldsymbol{\alpha}_{2}\right) \boldsymbol{i}-\cos \left(\boldsymbol{\theta}_{2}+\boldsymbol{\alpha}_{2}\right) \boldsymbol{k}\right]
$$

where: $M_{2 y}$ - moment of the slewing platform drive, $\alpha_{2}$ - position angle of the platform drive, $\theta_{2}$ - platform slewing angle, $D_{25}$ - diameter of the toothed ring of the axial bearing of the platform, $d_{24}$ - gear diameter on the drive output shaft.

The resulting force of possible digging resistance $W_{m}$ (Fig. 1a) acts on the cutting edge of the bucket and comprises component $W_{x y m}$ normal to the cutting edge and lateral component $W_{z m}$ collinear with the cutting edge of the bucket:

$$
\mathrm{W}_{m}=\sqrt{W_{x y m}^{2}+W_{z m}^{2}}
$$

whose direction is determined by the unit vector: 


$$
\begin{gathered}
\operatorname{ort} \boldsymbol{W}_{m}=\left(\cos \boldsymbol{\varphi}_{w m} \cos \boldsymbol{\varphi}_{w} \cos \boldsymbol{\theta}_{2}-\sin \boldsymbol{\varphi}_{w} \sin \boldsymbol{\theta}_{2}\right) \boldsymbol{i}+\cos \boldsymbol{\varphi}_{w m} \sin \boldsymbol{\varphi}_{w} \boldsymbol{j}+ \\
+\left(\cos \boldsymbol{\varphi}_{w} \cos \boldsymbol{\varphi}_{w m} \sin \boldsymbol{\theta}_{2}+\sin \boldsymbol{\varphi}_{w m} \cos \boldsymbol{\theta}_{2}\right) \boldsymbol{k}
\end{gathered}
$$

where: $\varphi_{w m}$ - angle between the direction of the action of the possible force of digging resistance $W_{m}$ and the force of the digging resistance normal to the cutting edge of bucket $W_{x y m}$, determined by the equation:

$$
\boldsymbol{\varphi}_{w m}=\operatorname{arctg} \frac{W_{z m}}{W_{x y m}}
$$

The intensity of the possible force of the digging resistance applied to the cutting edge of the bucket is

$$
W_{x y m}=\min \left\{W_{s m}, W_{p m}, W_{3 m}, W_{4 m}, W_{5 m}\right\}
$$

where: $W_{s m}, W_{s m}$ - boundary forces of the digging resistance that limit the stability of the excavator, $W_{3 m}, W_{4 m}, W_{5 m}$ - boundary forces of the digging resistance that can be overcome by the drive mechanisms of the manipulator boom, stick, and bucket.

The direction of the normal component of the possible force of the digging resistance $W_{x y m}$ in relation to the horizontal $O X Z$ plane of the absolute coordinate system is defined by the angle:

$$
\varphi_{w}=\varphi_{5}+\theta_{w}
$$

where: $\theta_{w}$ - angle of the digging resistance in relation to the positive $O_{5} x_{5}$ axis of the local coordinate system of bucket $L_{5}$.

The direction of the normal component of digging resistance $\boldsymbol{W}_{x y m}$ in the absolute coordinate system is determined by the unit vector:

$$
\text { ort } \boldsymbol{W}_{x y m}=\cos \boldsymbol{\varphi}_{w} \cdot \cos \boldsymbol{\theta}_{2} \boldsymbol{i}+\sin \boldsymbol{\varphi}_{w} \boldsymbol{j}+\sin \boldsymbol{\theta}_{2} \boldsymbol{k}
$$

The boundary force of digging resistance $W_{s m}$ that is limited by the static stability of the excavator is determined, depending on the position of the kinematic chain of the excavator and ort $W_{x y m}$, from the equilibrium conditions for one of the possible rotary joints $O_{11}, O_{12}$ between the support and movement member and the support surface, whose axes represent the potential excavator rollover lines (Fig. 1a) [17]:

$$
W_{s m}= \begin{cases}W_{s m 11}=\frac{-M_{o 11}}{\left(\left(\boldsymbol{r}_{w}-\boldsymbol{r}_{11}\right) \times o r t \boldsymbol{W}_{x y m}\right) \cdot \boldsymbol{e}_{1}}, & \begin{cases}\forall y_{w}>0, & \varphi_{12}>\varphi_{w}>\varphi_{11}+\pi \\ \forall y_{w}<0, & \varphi_{11}>\varphi_{w}>\varphi_{12}-\pi\end{cases} \\ W_{s m 12}=\frac{-M_{o l 2}}{\left(\left(\boldsymbol{r}_{w}-\boldsymbol{r}_{12}\right) \times o r t \boldsymbol{W}_{x y m}\right) \cdot \boldsymbol{e}_{1}}, & \begin{cases}\forall y_{w}>0, & \varphi_{12}+\pi>\varphi_{w}>\varphi_{11} \\ \forall y_{w}<0, & \varphi_{11}-\pi>\varphi_{w}>\varphi_{12}\end{cases} \end{cases}
$$

where: $M_{s m l 1}, M_{s m 12}$ - gravitational moments for longitudinal $x$ - $x$ or transverse $z-z$ excavator rollover line of the excavator, $\boldsymbol{r}_{11}, \boldsymbol{r}_{12}$ - vectors of the position of the center of the appropriate first rotary joint $O_{11}, O_{12}, \boldsymbol{e}_{1}$ - unit vector of the first rotary joint $O_{11}, O_{12}$, $\varphi_{11}, \varphi_{12}$ - angles of the position center of the cutting edge of the bucket in relation to the corresponding rotary joints defined by the equations:

$$
\boldsymbol{\varphi}_{11}=\arccos \left(\frac{\left(\boldsymbol{r}_{w}-\boldsymbol{r}_{11}\right) \cdot \boldsymbol{i}}{\left|\boldsymbol{r}_{w}-\boldsymbol{r}_{11}\right|}\right) \boldsymbol{\varphi}_{12}=\arccos \left(\frac{\left(\boldsymbol{r}_{w}-\boldsymbol{r}_{12}\right) \cdot \boldsymbol{i}}{\left|\boldsymbol{r}_{w}-\boldsymbol{r}_{12}\right|}\right)
$$


The gravity moments of the kinematic chain of the excavator for calculating possible longitudinal $x-x$ or transverse $z-z$ excavator rollover line are:

$$
M_{o l}=\left\{\begin{array}{l}
M_{o 11}=-g \sum_{k=1}^{k=5} m_{k}\left(\left(\boldsymbol{r}_{t k}-\boldsymbol{r}_{11}\right) \times \boldsymbol{j}\right) \cdot \boldsymbol{e}_{1}-g \sum_{k=3}^{k=5} m_{c k}\left(\left(\boldsymbol{r}_{c t k}-\boldsymbol{r}_{11}\right) \times \boldsymbol{j}\right) \cdot \boldsymbol{e}_{1}-g m_{z}\left(\left(\boldsymbol{r}_{t 5}-\boldsymbol{r}_{11}\right) \times \boldsymbol{j}\right) \cdot \boldsymbol{e}_{1} \\
M_{o l 2}=-g \sum_{k=1}^{k=5} m_{k}\left(\left(\boldsymbol{r}_{t k}-\boldsymbol{r}_{12}\right) \times \boldsymbol{j}\right) \cdot \boldsymbol{e}_{1}-g \sum_{k=3}^{k=5} m_{c k}\left(\left(\boldsymbol{r}_{c t k}-\boldsymbol{r}_{12}\right) \times \boldsymbol{j}\right) \cdot \boldsymbol{e}_{1}-g m_{z}\left(\left(\boldsymbol{r}_{t 5}-\boldsymbol{r}_{12}\right) \times \boldsymbol{j}\right) \cdot \boldsymbol{e}_{1}
\end{array}\right.
$$

where: $\boldsymbol{r}_{c t k}$ - vector of the position of the center mass of the hydraulic cylinder, $m_{z}$ - mass of the material scooped with the bucket.

Depending on the bucket position, the weight of material is:

$$
m_{z}=\left\{\begin{array}{lll}
\rho_{\mathrm{z}} \mathrm{V}\left|\cos \varphi_{5}\right| & \forall & 270^{\circ}<\varphi_{5}<90^{\circ} \\
0 & \forall & 270^{\circ} \geq \varphi_{5} \geq 90^{\circ}
\end{array}\right.
$$

where: $\rho_{z}$ - density of the material, $V$ - volume of the bucket.

From the non-sliding conditions of the excavator, i.e. the support and movement mechanism, in relation to the support surface during the digging operation and due to the action of the digging resistance force, the boundary of the digging resistance force $\mathrm{Wpm}$ was determined according to the size of the adhesion force occurring between the support and movement mechanism and the support surface:

$$
W_{p m}=\frac{m g \cdot \mu_{p}}{\left|\cos \varphi_{w}\right|}
$$

where: $m$ - mass of the excavator, $\mu_{p}$ - coefficient of adherence of the excavator movement mechanism to the support surface.

The boundary forces of digging resistance $W_{i m}$ that can be overcome by the drive mechanisms of manipulator boom $(i=3)$ and bucket $(i=5)$, for the known ort $W_{m}$ and the position of the kinematic chain of the excavator, during the operation of the maximum torques of mechanism $M_{\text {pimax }}$, are determined from the equilibrium conditions for axial joints $O_{i}(i=3,5)$ of the manipulator:

$$
W_{i m}=\frac{-M_{\text {pimax }}-M_{o i}}{\left(\left(\boldsymbol{r}_{w}-\boldsymbol{r}_{i}\right) \times \text { ort } \boldsymbol{W}_{x y m}\right) \cdot \boldsymbol{e}_{i}} \quad \forall i=3,5
$$

where: $M_{o i}$ - total moment of the gravitational forces of the kinematic chain members and the drive mechanisms of the excavator and the weight of the material scooped with the bucket for the individual axes of joints $O_{i}$.

The total moment of the excavator gravitational forces is determined by a set of moments:

$$
M_{o i}=M_{o i g}+M_{o i c}+M_{o i z} \quad \forall i=3,4,5
$$

where: $M_{\text {oig }}$ - moment of the gravitational forces of the members of the excavator kinematic chain for the individual axes of the joints $O_{i}, M_{\text {oic }}$ - moment of the gravitational forces of the members of the excavator drive mechanisms, $M_{o i z}$ - moment of the weight of the material for the individual axes of joints $O_{i}$. The moment of the gravitational 
forces of the members of the excavator kinematic chain for individual axes of joints $O_{i}$, is determined by the equation:

$$
M_{\text {oig }}=-g \sum_{k=1}^{k=5} m_{k}\left(\left(\boldsymbol{r}_{t k}-\boldsymbol{r}_{i}\right) \times \boldsymbol{j}\right) \cdot \boldsymbol{e}_{i} \quad \forall i=3,4,5
$$

where: $\boldsymbol{e}_{i}$ - unit vector of joint axis $O_{i}$.

The moments of the gravitational forces of the members of the excavator drive mechanisms for individual axes of joints $O_{i}$ are determined by the following equations $[18,19]$ :

$$
M_{o i c}=\left\{\begin{array}{l}
M_{o c 3}=-g \frac{n_{c 3} m_{c 3}}{2}\left(\left(\boldsymbol{r}_{A 3}-\boldsymbol{r}_{3}\right) \times \boldsymbol{j}\right) \cdot \boldsymbol{e}_{3}-g \sum_{k=4}^{k=5} n_{c k} m_{c k}\left(\left(r_{c k}-r_{3}\right) \times j\right) \cdot e_{3} \quad \forall i=3 \\
M_{o c 4}=-g \frac{n_{c 4} m_{c 4}}{2}\left(\left(\boldsymbol{r}_{A 4}-\boldsymbol{r}_{4}\right) \times \boldsymbol{j}\right) \cdot \boldsymbol{e}_{4}-g \frac{n_{c 5} m_{c 5}}{2}\left(\left(r_{A 5}-r_{4}\right) \times j\right) \cdot \boldsymbol{e}_{4} \quad \forall i=4 \\
M_{o c 5}=-g \frac{m_{c 5}}{2}\left(\left(\boldsymbol{r}_{A 5}-\boldsymbol{r}_{5}\right) \times \boldsymbol{j}\right) \cdot \boldsymbol{e}_{5} \quad \forall i=5
\end{array}\right.
$$

where: $\boldsymbol{r}_{A 3}, \boldsymbol{r}_{A 4,} \boldsymbol{r}_{A 5}$ - coordinates of the joints in which the hydraulic cylinders are linked to the members of the kinematic chain (Fig. 2).

The moment of the material weight for the individual axes of joints $O_{i}$ is determined by the equation:

$$
M_{\text {oiz }}=-g m_{z}\left(\left(\boldsymbol{r}_{t 5}-\boldsymbol{r}_{r}\right) \times \boldsymbol{j}\right) \cdot \boldsymbol{e}_{i} \quad \forall i=3,4,5
$$

where: $m_{z}$ - mass of the material scooped with the bucket, assuming that the center of mass coincides with the bucket mass center.

The maximum drive moments of manipulator mechanisms for both directions in operation (upon piston pushing and piston pulling in the hydraulic cylinder):

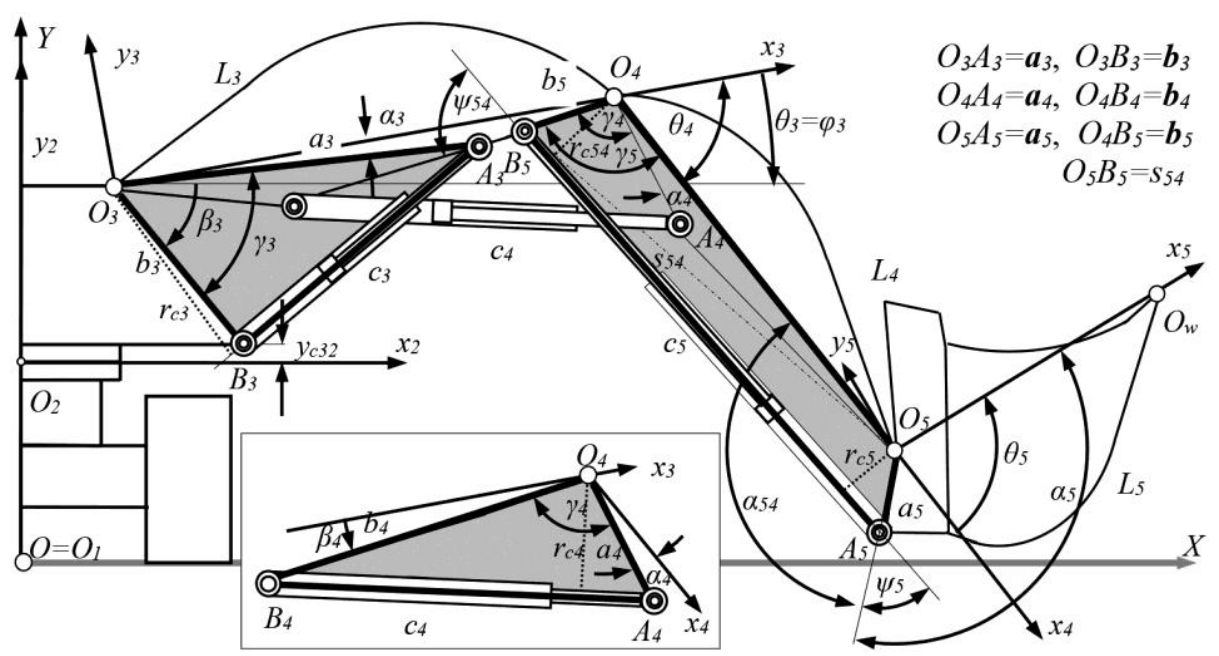

Fig. 2 Models of drive mechanisms of the excavator with a loading manipulator 


$$
M_{\text {pimax }}=\left\{\begin{array}{l}
M_{p i l \max }=\operatorname{sign}\left(\dot{\theta}_{i}\right) \cdot r_{c i} \cdot n_{c i} \frac{d_{i 1}^{2} \pi}{4} p_{\max } \quad \forall i=3,4,5 \quad \dot{\theta}_{3}>0, \dot{\theta}_{4}>0, \dot{\theta}_{5}>0 \\
M_{p i 2 \max }=\operatorname{sign}\left(\dot{\theta}_{i}\right) \cdot r_{c i} \cdot n_{c i} \frac{\left(d_{i 1}^{2}-d_{i 2}^{2}\right) \pi}{4} p_{\max } \quad \forall i=3,4,5 \quad \dot{\theta}_{3}<0, \dot{\theta}_{4}<0, \dot{\theta}_{5}<0
\end{array}\right.
$$

where: $r_{c i}$ - transmission function of the drive mechanism of member $L_{i}, \dot{\boldsymbol{\theta}}_{i}$ - angular velocity of the active member of the kinematic pair mechanism around the axis of joint $O_{i}, p_{\max }$ - maximum pressure of the hydrostatic excavator system.

The transmission function of the drive moment of the boom, stick and bucket mechanisms for the axis of joint $\mathrm{O}_{3}, \mathrm{O}_{4}, \mathrm{O}_{5}$ (Fig. 2):

$$
\begin{gathered}
r_{c 3}=a_{3} \frac{b_{3}}{c_{3}} \sin \gamma_{3}=a_{3} \frac{b_{3}}{c_{3}} \sin \left(\arccos \frac{b_{3}^{2}+a_{3}^{2}-c_{3}^{2}}{2 a_{3} b_{3}}\right), \\
r_{c 4}=a_{4} \frac{b_{4}}{c_{4}} \sin \gamma_{4}=a_{4} \frac{b_{4}}{c_{4}} \sin \left(-\arccos \frac{b_{4}^{2}+a_{4}^{2}-c_{4}^{2}}{2 b_{4} \cdot a_{4}}\right), \\
r_{c 5}=a_{5} \sin \psi_{5}=a_{5} \sin \left(\arccos \frac{a_{5}^{2}+c_{5}^{2}-s_{54}^{2}}{2 a_{5} c_{5}}\right)
\end{gathered}
$$

where: $s_{54}=b_{5}^{2}+s_{4}^{2}-2 b_{5} \cdot s_{4} \cos \left(\gamma_{4}-\arccos \frac{\hat{\boldsymbol{a}}_{4} \cdot \hat{\boldsymbol{i}}_{4}}{\mathrm{a}_{4}}\right) a_{i}, b_{i}, c_{i}(i=3,4,5)$ - intensity of the position vector joints in which the hydraulic cylinder mechanisms are linked to the kinematic chain members and the length of the hydraulic cylinders, $s_{4}$ - kinematic length of the stick.

The boundary force of digging resistance $W_{4 m}$ that can be overcome by the drive mechanism of the manipulator stick, for the known ort $W_{x y m}$ and the position of the excavator kinematic chain, during the operation of maximum drive moment $M_{p 4 \max }$, is determined from the condition of equilibrium for the axis of joints $O_{4}$ and $O_{5}$ of the manipulator because the stick and bucket mechanisms are dependent mechanisms (Fig. 2):

$$
W_{4 m}\left(\left(\boldsymbol{r}_{w}-\boldsymbol{r}_{4}\right) \times \text { ort } \boldsymbol{W}_{x y m}\right) \cdot \boldsymbol{e}_{4}+F_{c 54} \cdot r_{c 54}+M_{o 4}+M_{p 4 \max }=0
$$

where: $r_{c 54}$ - transmission function of the drive moment of the bucket mechanism for the axis of joint $\mathrm{O}_{4}, F_{c 54}$ - force in the hydraulic cylinder of the bucket subjected to boundary resistance $W_{4 m}$.

The transmission function of the drive moment of the bucket mechanism for the axis of joint $\mathrm{O}_{4}$ is determined by equation [20]:

$$
i_{c 54}^{M}=r_{c 54}=b_{5} \sin \psi_{54}
$$

where: $\boldsymbol{\psi}_{54}=\arccos \frac{s_{54}^{2}+b_{5}^{2}-s_{4}^{2}}{2 s_{54} b_{5}}+\arccos \frac{s_{54}^{2}+c_{5}^{2}-a_{5}^{2}}{2 s_{54} c_{5}}$

From the condition of equilibrium for joint $O_{5}$ when the boundary force of digging resistance $W_{4 m}$ acts:

$$
W_{4 m}\left(\left(\boldsymbol{r}_{w}-\boldsymbol{r}_{5}\right) \times \operatorname{ort} \boldsymbol{W}_{x y m}\right) \cdot \boldsymbol{e}_{5}+F_{c 54} \cdot r_{c 5}+M_{o 5}=0
$$


the force in the bucket hydraulic cylinder is determined:

$$
F_{c 54}=\frac{-W_{4 m}\left(\left(\boldsymbol{r}_{w}-\boldsymbol{r}_{5}\right) \times \operatorname{ort} \boldsymbol{W}_{x y m}\right) \cdot \boldsymbol{e}_{5}-M_{o 5}}{r_{c 5}}
$$

By substituting force $F_{c 54}$ in Eq. (30), boundary digging resistance $W_{4 m}$ was obtained:

$$
W_{4 m}=\frac{-r_{c 5}\left(M_{p 4 \max }+M_{o 4}\right)+r_{c 54} M_{o 5}}{r_{c 5}\left(\left(\boldsymbol{r}_{w}-\boldsymbol{r}_{4}\right) \times \boldsymbol{o r t} \boldsymbol{W}_{x y m}\right) \cdot \boldsymbol{e}_{4}-r_{c 54}\left(\left(\boldsymbol{r}_{w}-\boldsymbol{r}_{5}\right) \times \operatorname{ort} \boldsymbol{W}_{x y m}\right) \cdot \boldsymbol{e}_{5}}
$$

Depending on the moment of rotation of the tracked support and movement mechanism in relation to the surface and the operation of the horizontal component of the normal force of the possible digging resistance, the component of the possible force of the digging resistance, collinear with the cutting edge of the bucket, is determined by the following equation:

$$
W_{z m}=\left(\mu_{o} \frac{m g \cdot L}{4}+W_{x m} \cdot z_{w}\right) \cdot \frac{1}{x_{w}}
$$

where: $\mu_{o}$ - coefficient of the turning resistance of the tracks against the excavator support surface, $L$ - length of the continuous tracks footprint, $W_{x m}$-component of the possible digging resistance, $x_{w}, z_{w}$ - coordinates of the action of the possible digging resistance on the cutting edge of the bucket.

The size of axial bearings of the excavator slewing platform is determined on the basis of the equivalent loads defined by the bearing manufacturers in the form (Fig. 1b) [21]:

- for equivalent force: $\quad F_{e s}=\left(a \cdot F_{2 a}+b \cdot F_{2 r}\right) \cdot f_{s}$

- for equivalent moment: $\quad M_{e s}=c \cdot M_{2 r} \cdot f_{s}$

where: $F_{2 a}, F_{2 r}$ - axial and radial force of the axial bearing loading, $M_{2 r}$ - moment of the axial bearing loading, $a, b, c$-coefficients depending on the type of bearing, the type and size of the machine and its working conditions, $f_{s}$ - factor of static safety.

\section{ANALYSIS}

The analysis of the influence of the position and digging resistance in the entire working area on the change in the equivalent load of the axial bearing of the excavator slewing platform was carried out on an excavator of the weight of $m=100000 \mathrm{~kg}$ and with the loading manipulator bucket of $V=6,5 \mathrm{~m}^{3}$ in volume. The analysis used the hodographs of boundary and possible digging forces and the spectra of axial bearing loads of the slewing platform drive mechanism defined for certain segments of the entire working area of the excavator.

\subsection{Analysis of the impact of possible forces of digging resistance}

Using the developed program based on the defined mathematical model of the excavator, the $H W_{i}$ (Fig.3) and possible $\left(H W_{m}\right)$ resistance forces for two different positions of the excavator kinematic chain are defined: $\varphi_{3}=21,51^{\circ}, \varphi_{4}=-99,74^{\circ}, \varphi_{5}=-91,83^{\circ}$ (Fig. 3a), $\varphi_{3}=9,58^{\circ}, \varphi_{4}=-54,52^{\circ}, \varphi_{5}=-61,83^{\circ}$ (Fig. 3b). 
For a specified position of the kinematic chain, the hodograph of the boundary resistance $\left(H W_{i m}\right)$ presents, in relation to the center of the cutting edge of bucket $O_{w}$, a line perpendicular to the vector of the boundary resistance $\left(W_{i m} \mathrm{~min}\right)$, of minimal value determined from the operation of the driving mechanism in both directions [22]. The hodograph of the potential digging resistance, in relation to the center of the cutting edge of bucket $O_{w}$, is a polygon bounded by the hodographs of the boundary digging resistances (forces) limited by the excavator stability and the hodographs of the boundary digging resistances (forces) limited by the manipulator drive mechanisms. Normal $n-n$ drawn on the current speed of digging $v$, divides the hodograph of the possible digging resistance (force) into: a) the zone with a possible natural direction of action corresponding to the digging technology, and b) the zone with a direction of action inappropriate to the digging technology.

For the two different positions of the excavator kinematic chain, the comparative analysis shows (Fig. 3a, b and Table 1) that the hodographs of the digging resistance forces vary considerably.

For different boom and stick positions, the same bucket position $\left(\varphi_{5}=-61.83^{\circ}\right)$ and the same direction of the digging resistance force $\left(\varphi_{w}=238.17^{\circ}\right)$, which corresponds to the excavator digging technology with the shovel bucket, the intensity of the possible digging resistance in the first position is equal to the boundary force of the digging resistance that can be overcome by the bucket mechanism $\left(W_{m}=W_{5 m}\right)$. In the second position, the possible digging resistance is considerably lower in relation to the first position and is limited by the stability of the excavator $\left(W_{m}=W_{s m}\right)$.
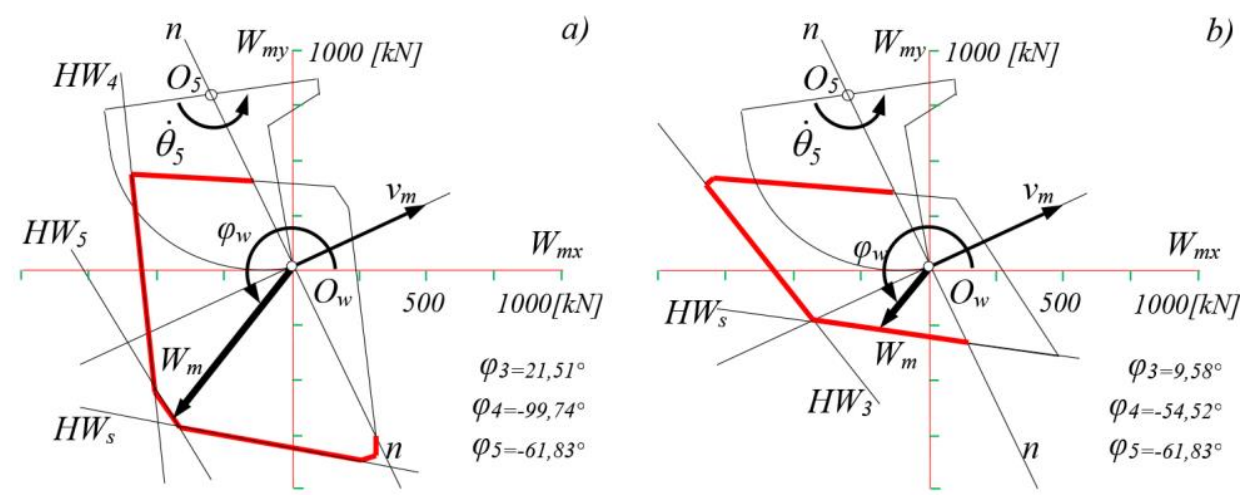

Fig. 3 Hodographs of possible digging forces (resistances) for two different positions of the excavator kinematic chain

There is also a difference in the size of the equivalent loads $\left(F_{e s}, M_{e s}\right)$ of the axial bearing due to the difference in the position of the excavator kinematic chain and the difference in the possible forces of digging resistance.

The obtained results point to a complex problem of determining the load of the axial bearing of the slewing platform drive mechanism since it has many different positions of the kinematic chain during operation throughout the entire working area; moreover, there are many different possible forces of digging resistance in every position. 
Table 1 The position and load parameters of the excavator valid for the choice of axial bearing

\begin{tabular}{|c|c|c|c|}
\hline \multirow{2}{*}{ Name and symbol of quantities } & \multirow{2}{*}{ Dimension- } & \multicolumn{2}{|c|}{ Value } \\
\hline & & position I & position II \\
\hline Horizontal reach, $x_{w}$ & $m$ & 5,30 & 8,09 \\
\hline Vertical reach, $y_{w}$ & $m$ & $-0,64$ & $-0,99$ \\
\hline Position angle of boom, $\varphi_{3}$ & $o$ & 21,51 & 9,58 \\
\hline Position angle of stick, $\varphi_{4}$ & $o$ & $-99,74$ & $-54,52$ \\
\hline Position angle of bucket, $\varphi_{5}$ & $o$ & $-61,83$ & $-61,83$ \\
\hline Angle of action force of digging resistance, $\varphi_{w}$ & $o$ & 238,17 & 238,17 \\
\hline Possible force of digging resistance, $W_{m}$ & $k N$ & 823,24 & 326.889 \\
\hline Boundary digging resistance force allowed by stability, $W_{s m}$ & $k N$ & 841,22 & 326.889 \\
\hline Boundary digging resistance force of boom mechanism, $W_{3 m}$ & $k N$ & 835,99 & 497,46 \\
\hline Boundary digging resistance force of stick mechanism, $W_{4 m}$ & $k N$ & 928,42 & 538,30 \\
\hline Boundary digging resistance force of bucket mechanism, $W_{5 m}$ & $k N$ & 823,24 & 641,32 \\
\hline Equivalent force, $F_{e s}$ & $k N$ & 3325,76 & 1934,83 \\
\hline Equivalent moment, $M_{e s}$ & $k N m$ & 6815,27 & 3916,97 \\
\hline
\end{tabular}

\subsection{Analysis of the impact of the digging position}

In order to analyze the influence of the change in the position of the kinematic chain of the excavator, i.e. the change in the digging position throughout the entire working area, on the change in the equivalent loads of the slewing platform axial bearing, the axial bearing spectrum load was used. The spectrum of the axial bearing equivalent loads was determined using the developed program by spatial simulation - by changing the position of each member of the manipulator kinematic chain, in its range of movement for 60000 different positions of the manipulator kinematic chain, i.e. possible digging positions in the entire working area of the excavator of the weight of $100000 \mathrm{~kg}$ and a loading manipulator with the bucket of $6,5 \mathrm{~m}^{3}$ in volume.

The obtained spectrum of axial bearing loads is shown in the diagram (Fig. 4a) $(A L 3, \ldots, A L 6)$, which presents the dependence of the allowed values of equivalent moments of $M_{e s}$ and equivalent forces $F_{e s}$ of axial bearing loads. In order to find the position of the kinematic chain in which the equivalent loads arise for the choice of the axial bearing size, the entire working area of the excavator is divided into four segment fields (Fig.4b) and the equivalent loads of the axial bearing of the slewing platform mechanism are determined for each segment field.

The spectra of the equivalent load of axial bearings for each segment field of operation are shown, in appropriate colors, in the bearing diagrams of the available axial bearings. A comparison of the spectra shows that the bearing loads are very different in segment fields. According to the equivalent loads in the segment fields, the slewing platform mechanism corresponds to the different sizes of the available axial bearings. The position of the kinematic chain of the excavator in which the corresponding equivalent loads for the choice of the axial bearing size are found, is determined using the developed program, on the condition that the point, from the constellation of the spectra of the equivalent bearing load, is the least removed from the allowed load bearing capacity of selected bearing size $A L 6$. In the isolated position of the excavator kinematic chain, when the corresponding bearing loads are applied, according to the hodograph of the possible forces of digging resistance (Fig. 3), the boundary forces of digging resistance have approximately the same values (Table 1), and the force of the possible digging resistance has the direction which belongs to the zone with the possible natural action directions that correspond to the digging technology. 
The analysis shows that the set of equivalent loads, which are close to the equivalent bearing loads, corresponds to the positions (Fig. 4c) of the excavator kinematic chain in the zone of the working area when the manipulator drive mechanisms have such positions and coordinated interaction that they can overcome the largest digging resistance forces allowed by the excavator stability.
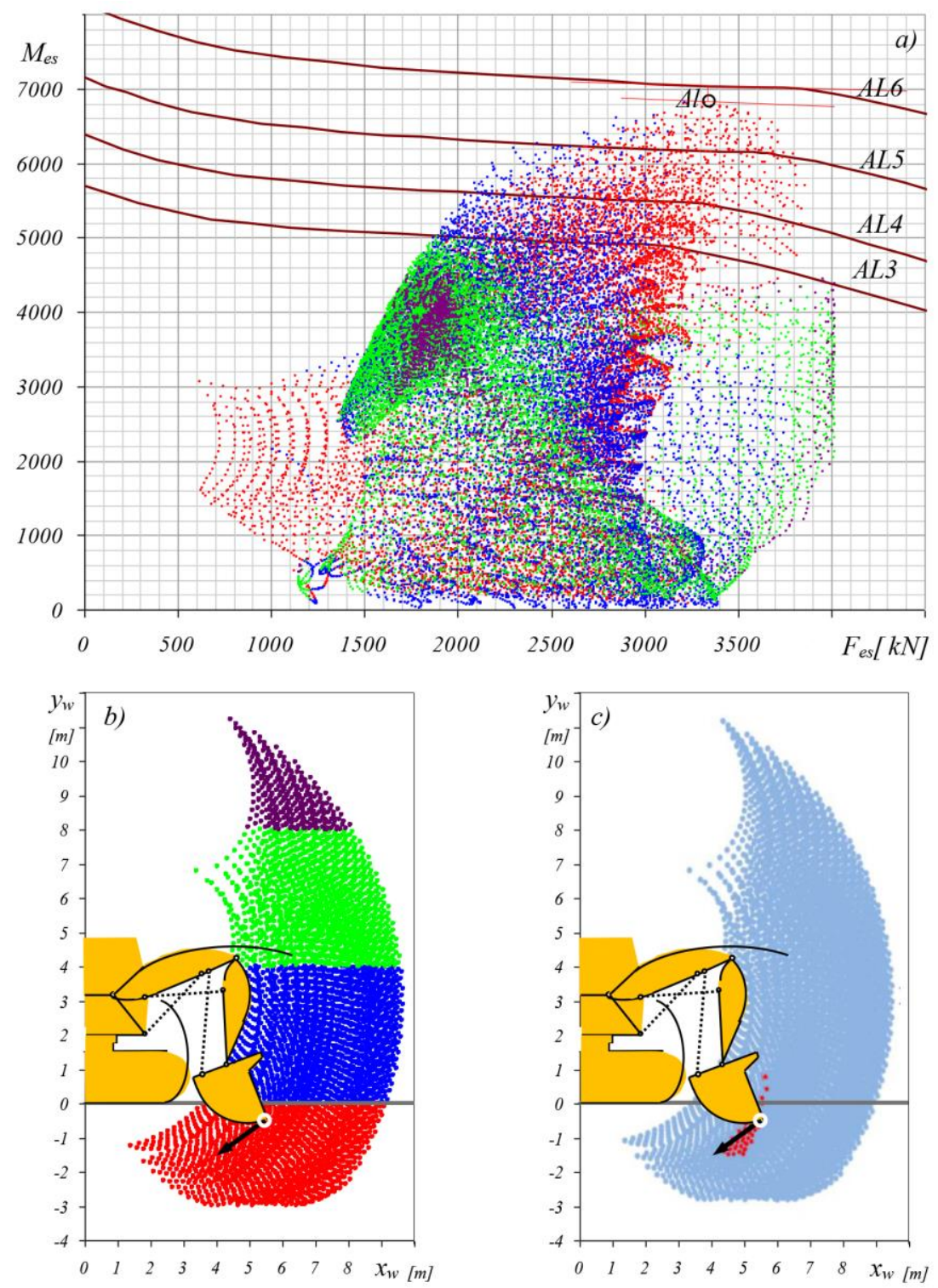

Fig. 4 Analysis of axial bearing load of slewing platform drive depending on the position of the loading manipulator of excavator: a) equivalent loads in the entire working area, b) segmental fields of the working area of the excavator, and c) area of relevant loads 


\section{CONCLUSION}

The choice of the axial bearing size in the slewing platform drive mechanism in hydraulic excavators is very complex due to a large number of different impacts on the bearing load. One of the more important impacts is the digging position in the excavator's working area. Hydraulic excavators are distinguished by the ability to perform various functions with various tools (bucket, grapple, hook, grabber). It is characteristic that in carrying out any possible functions in their working area, hydraulic excavators have many different positions and working conditions. Using the developed mathematical model and program, the paper analyzes the effects of the digging position on the load of the axial bearing of the slewing platform drive mechanism in an excavator equipped with a shovel bucket.

The analysis uses the hodographs of the boundary forces of digging resistance and the axial bearing load spectra. The hodographs of the digging resistance forces show that the possible forces of digging resistance, as a primary load of the axial bearing, are very variable and depend on the digging position or the position of the excavator kinematic chain. Using the axial bearing load spectrum, the working area fields are determined - i.e. the position of the excavator kinematic chain, and the vectors and the hodographs of the digging resistance forces, as well as the possible digging resistance vectors, which yield the equivalent loads relevant to the choice of the axial bearing of the slewing platform drive mechanism in hydraulic excavators.

Acknowledgements: This paper presents the results of the research conducted within the project "Research and Development of New Generation Machine Systems in the Function of the Technological Development of Serbia" funded by the Faculty of Mechanical Engineering, University of Niš, Serbia.

\section{REFERENCES}

1. Jiaqi, X., Hwan-Sik, Y., 2016, A review on mechanical and hydraulic system modeling of excavator manipulator system, Journal of Construction Engineering, 2016, pp. 1-11.

2. Mitrev, R., Janošević, D., Marinković, D., 2017, Dynamical modelling of hydraulic excavator considered as a multibody system, Tehnički Vjesnik, 24, pp. 831-836.

3. Vujić, D., Lazarević, O., Batinić, V., 2017, Development of dynamic-mathematical model of hydraulic excavator, Journal of Central South University, 24, pp. 2010-2018.

4. Mitrev, R., Marinkovic, D., 2019, Numerical study of the hydraulic excavator overturning stability during performing lifting operations, Advances in Mechanical Engineering, 11(5), doi: 10.1177/1687814019841779.

5. Hua, W., Peiyu, H., Bitao, P., Xuehai, G., 2017, A new computational model of large three-row roller slewing bearings using nonlinear springs, Proc. Institution of Mechanical Engineers, Part C: Journal of Mechanical Engineering Science, 231(20), pp. 3831-3839.

6. Smolnicki, T., Stańco, M., Derlukiewicz, D., 2013, Distribution of loads in the large size bearing - problems of identification, Tehnički vjesnik, 20(5), pp. 831-836.

7. Aguirrebeitia, J., Abasolo, M., Aviles, R., Fernandez, I., 2011, General static load capacity in slewing bearings. Unified theoretical approach for crossed roller bearings and four contact point angular ball bearings, 13th World Congress in Mechanism and Machine Science, Guanajuato, México 19-25 June, A19_362.

8. Krynke, M., Selejdak, J., Borkowski, S., 2013, Determination of static limiting load curves for slewing bearing with application of the finite element method, Materials Engineering - Materiálové inžinierstvo, 20, pp. 64-70.

9. Potočnik, R., Göncz, P., Glodež, S., 2013, Static capacity of a large double row slewing ball bearing with predefined irregular geometry, Mechanism and Machine Theory, 64, pp. 67-79.

10. Kania, L., Krynke, M., 2013, Computation of the general carrying capacity of slewing bearings, Engineering Computations, 30(7), pp. 1011-1028. 
11. Wang, Y., Yuan, Q., 2014, Static load-carrying capacity and fatigue life of a double row pitch bearing with radial interference, The Journal of Mechanical Engineering Science, 228(2), pp. 307-316.

12. Jovanović, V., 2018, A contribution to the synthesis of the slewing platform drive mechanism of hydraulic excavators, PhD dissertation, (in Serbian), University of Niš, Faculty of Mechanical Engineering, 186p.

13. Kagoshima, M., 2013, The development of an 8 tonne class hybrid hydraulic excavator SK80H, Kobelco Technology Review, 31, pp. 6-11.

14. Joo, C., Stangl, M., 2016, Application of power regenerative boom system to excavator, 10th International Fluid Power Conference, Dresden, Group 10 - Mobile Hydraulics, Paper 10-3, pp. 175-184.

15. Li, W., Cao, B., Zhu, Z., Chen, G., 2014, A novel energy recovery system for parallel hybrid hydraulic excavator, The Scientific World Journal, 2014, Article ID 184909, pp. 1-14.

16. Jovanović, V., Janošević, D., Petrović, N., 2014, Experimental determination of bearing loads in rotating platform drive mechanisms of hydraulic excavators, Facta Univesitatis-Series Mechanical Engineering, 12(2), pp. 157-169.

17. Jovanović, V., Janošević, D., Pavlović, J., Petrović, N., 2014, Definition of directed digging force for assessment of the hydraulic excavator work, The 8th International Symposium - KOD 2014, Faculty of Tehnical Sciences, University of Novi Sad Slovak University of Technology in Bratislava International Federation for the Promotion of Mechanism and Machine Science - IFToMM Association for Design, Elements and Constructions - ADEKO, pp. 51-54.

18. Janošević, D., Pavlović, J., Jovanović, V., Petrović, G., 2018, A numerical and experimental analysis of the dynamic stability of hydraulic excavators, Facta Univesitatis-Series Mechanical Engineering, 16(2), pp. 157-170.

19. Jovanović, V., Janošević, D., Marinković, D., 2015, Determination of the load acting on the axial bearing of a slewing platform drive in hydraulic excavators, Acta Polytechnica Hungarica, 12(1), pp. 5-22.

20. Janošević, D., Jovanović, V., 2015, Synthesis of drive mechanisms of a hydraulic excavator, monograph, (in Serbian), University of Niš, Faculty of Mechanical Engineering.

21. Catalog thyssenkrupp Rothe Erde, Slewing bearings, 2007, GmbH D-44137 Dortmund.

22. Pavlović, J., Janošević, D., Jovanović, V., 2018, Optimization of a loader mechanism on the basis of the directed digging force, Iranian Journal of Science and Technology, Transactions of Mechanical Engineering, doi: 10.1007/s40997-018-0236-z. 